\title{
Harnessing Citizen Science to Assess and Improve Utilization of Metropolitan Parks: the Park Activity, Recreation, and Community Study (PARCS) in St. Louis, MO
}

\author{
Áine O'Connor ${ }^{1}$, Abby C. King ${ }^{2}$, Ann Banchoff ${ }^{2}$, Amy Eyler ${ }^{1}$, Rodrigo Reis ${ }^{1,3}$, Ross C. Brownson ${ }^{1,4}$, \\ Eugen Resendiz ${ }^{1}$, Derek Holland ${ }^{1}$, and Deborah Salvo ${ }^{1}$ \\ ${ }^{1}$ Washington University in St. Louis, Prevention Research Center, Brown School, USA \\ ${ }^{2}$ Stanford University, Stanford Prevention Research Center, USA \\ ${ }^{3}$ Pontifical Catholic University, Urban Management, Graduate Program, Brazil \\ ${ }^{4}$ Washington University in St. Louis, School of Medicine, Division of Public Health Sciences, and Alvin \\ J. Siteman Cancer Center, Department of Surgery, USA
}

\begin{abstract}
Access to and use of parks is associated with physical activity participation. Our Voice is a systematic method blending community-based participatory research (CBPR) and citizen science. As part of a comprehensive, mixed-methods study in St. Louis, Missouri (PARCS), we tested the feasibility of the Our Voice method for gathering community input on the barriers to and facilitators of accessibility and use of large metropolitan parks, by describing the implementation of the Our Voice method among recreational and commuter users of a large metropolitan park in St. Louis, MO. Due to challenges posed by COVID-19, the Our Voice methodology was adapted for remote participation. Twenty-three citizen scientists (14 recreational park users and 9 commuters) collected and analyzed geolocated route, photo, and audio or text data on facilitators and barriers to park use and access. They identified 6 priority themes and 12 solution ideas, and presented them to stakeholders. In contrast to previous $O u r$ Voice studies, separate user groups (recreation and commuter users) independently prioritized many of the same themes. Adaptation of the Our Voice protocol to virtual practices during COVID-19 revealed positive implications for cost, reach, and scale of studies grounded in CBPR and citizen science. We provide a set of recommended practices for using Our Voice as a method to evaluate and promote equity of access and use of metropolitan parks.
\end{abstract}

Keywords: built environment, citizen science, active living, health equity, urban green space 
By providing access to places and programs for active living, contact with nature, and social interaction, parks can be vital contributors to city residents' health and wellbeing (Bedimo-Rung et al., 2005; Ferdinand et al., 2012; Gelormino et al., 2015). Previous research indicates that urban park access promotes improvements in residents' physical activity, mental health, social engagement, and self-reported wellbeing and quality of life (Morgan Hughey et al., 2017; Rugel et al., 2019; Schipperijn et al., 2017; Wood et al., 2017). However, levels of park access and use vary across communities. Proximity to home, number of amenities offered, perceived safety, and the presence of incivilities, such as trash or graffiti, have each been linked to differences in utilization rates (Humpel et al., 2002; Kaczynski \& Henderson, 2007; Sallis et al., 2016). Ensuring that community members have equitable access to parks that are safe, clean, and provide diverse amenities for users can help promote health and wellbeing in urban areas.

Most evidence for the health benefits of urban parks comes from the study of residential-neighborhood-based spaces (small or medium parks and recreation centers) (Kaczynski \& Henderson, 2007; McCormack et al., 2010; Sallis et al., 2016). Large metropolitan parks, however, are unique in size, service area, and the diversity of their natural and built assets. In metropolitan parks that border multiple neighborhoods, linear elements like trails and sidewalks may provide residents with safe venues for active commuting through the city as well as leisure-time bicycling, walking, running, and other recreational activities. By providing plentiful natural green and blue spaces, metropolitan parks can contribute to improved mental health outcomes as well as environmental sustainability (Gascon et al., 2015; Jimenez et al., 2021; Vanaken \& Danckaerts, 2018; Wood et al., 2017; Jennings et al., 2016; Kruizse et al., 2019). Community gathering places in metropolitan parks like pavilions, picnic areas, and designated event spaces provide an excellent setting for formal and informal gatherings, which can help build social capital (Kaźmierczak, 2013; Leyden, 2003; Maas et al., 2009; Rugel et al., 2019). In addition, some iconic metropolitan parks (e.g., Central Park in New York City and Forest Park in St. Louis, USA; Ibirapuera Park in São Paulo, Brazil; Hyde Park in London, UK) are host to a number of cultural assets including zoos, museums, and exhibition centers.

Another unique aspect of metropolitan parks is their organizational and administrative complexity. Multiple municipal and regional departments including parks and recreation, natural resources or forestry, transportation, culture and tourism, economic development, or others, are often involved in different capacities in these types of parks. Nongovernmental organizations (e.g., foundations, citizen-led advocacy groups, etc.) may also support different aspects of the park or areas/institutions within it. Responsibility for decision-making regarding changes to park amenities or design is often shared between these stakeholders.
In addition to focusing on smaller parks, most public health-driven studies of park access and use rely on traditional data collection and analysis methods. These include direct observation techniques such as the System for Observing Play and Recreation in Communities (SOPARC), park user or stakeholder surveys or interviews, and environmental audits using assessment tools such as the Environmental Assessment of Public Recreation Spaces (EAPRS) and the Physical Activity Resource Assessment (PARA) (McKenzie et al., 2016; Saelens et al., 2006; Lee et al., 2005). Environmental audits consist of standardized checklists or inventories of parks' physical properties, such as the availability, quality, and condition of facilities and amenities. While valuable, when used alone, these methods lack sufficient social context to prioritize issues of importance for community members and stakeholders. In the case of environmental audits, another weakness is that they tend to be very lengthy and time-consuming, and as such, they are not usually feasible options for assessing large and amenity-rich urban metropolitan parks. By contrast, community-based participatory research (CBPR) and citizen science provide community-driven approaches to data collection and analysis that present unique advantages for understanding drivers of use, barriers and facilitators of access, and priorities for improving community assets like metropolitan parks. CBPR intentionally shapes research practices around the engagement of community members at every stage of a study, with the dual purposes of gathering grassroots, livedexperience knowledge about population health and building capacity with community partners (Israel, Schultz, et al., 1998; Israel, Coombe, et al., 2010). Similarly, citizen science engages community volunteers - who may or may not possess training in scientific research - to systematically collect and potentially analyze data in research studies (Paulos et al., 2008). Citizen scientist participation can be classified on a continuum, from "for the people" methods of crowdsourcing or donating information for others to analyze to "by the people" approaches in which residents collect information about their own environments and are provided with instruction on using those data for positive local change and broader community benefit (King, Winter, Sheats, et al., 2016; King, Winter, Chrisinger, et al., 2019).

The Our Voice Citizen Science Engagement Model (Our Voice) is a "by the people" approach, combining CBPR and citizen science by inviting community members to be trained in data collection, analysis, and community advocacy for the express purpose of changing local environments based on their own priorities. One unique aspect of Our Voice is that it incorporates data and participation by local residents as well as input from key stakeholders of multiple sectors to reach agreements and prioritize action. Our Voice has been successfully used in projects worldwide to assess and improve food access, safe routes to school, and neighborhood environments of diverse populations across the life course and for different purposes (e.g., healthy eating, active living, aging-in-place, etc.) (Buman, Bertmann, et al., 2015; Chrisinger et al., 2018; Sheats et al., 2017; González et al., 2021; Rodriguez et al., 
2019; Moran et al., 2017; Tuckett, Banchoff, et al., 2018; Tuckett, Freeman, et al., 2018; King, King, et al., 2020; King, Odunitan-Wayas, et al., 2021). The Our Voice model has also been implemented to evaluate community access to and use of parks, including local neighborhood parks in Colombia and a small pop-up park in Los Altos, California ( Rubio et al., 2021; Salvo et al., 2017; Winter, Sheats, et al., 2020). However, this method has not previously been used within the context of large metropolitan parks.

The aims of this study were to (a) test the feasibility of the Our Voice method for gathering community input on the barriers and facilitators of accessibility and use of large metropolitan parks; (b) describe the implementation of the Our Voice method among recreational and commuter users of a large metropolitan park in St. Louis, MO, and (c) present recommendations for use of the Our Voice method to promote equity in access to major health- and wellbeingenhancing city resources, like metropolitan parks.

\section{Methods}

\section{Parent Study and Setting}

The Park Activity, Recreation, and Community Study (PARCS) is a mixed-methods study characterizing the patterns and determinants of use of a 1,300-acre metropolitan park in St. Louis, Missouri. The park is the largest in the region and is centrally located in St. Louis' 15-county metropolitan area, often defined as the service area of the park. St. Louis City, which operates the park jointly with a nonprofit conservancy, is an independent municipality governed separately from adjacent St. Louis County (see Appendix for Figure 1). Although the park is in St. Louis City, its western edge lies on the boundary between the two municipalities. St. Louis City has 300,000 residents: $45 \%$ are Black and $4 \%$ Hispanic or Latino; $36 \%$ have a 4-year college degree. By contrast, St. Louis County is a majority (67.9\%) white area of 994,000 residents, $43.6 \%$ of whom have a 4 -year degree. The greater St. Louis metropolitan area is a majority-white region of 2.8 million residents that spans counties in Missouri and Illinois.

The parent study is still ongoing as of November 2021, and data is being collected through a variety of methods. This report summarizes the experience and findings of the first cohort of citizen scientists, all frequent park users, who completed the Our Voice process as part of PARCS. The second PARCS Our Voice phase will recruit citizen scientists from park-proximate neighborhoods with low rates of park use, engaging a cross-section of the greater St. Louis community.

\section{Our Voice Citizen Science Engagement Model}

Our Voice uses mobile technology to engage community members as catalysts for change in their local environments through a four-step approach (King, Winter, Sheats, et al., 2016).

1. Discover. Residents train as citizen scientists, using a mobile application (the Stanford Healthy
Neighborhood Discovery Tool, or "Discovery Tool") to collect data on the features of their environment that help or hinder health behaviors and wellbeing (Buman, Winter, et al., 2013). The Discovery Tool, which can be downloaded from either the Apple App or Google Play stores, allows citizen scientists to capture geocoded photographs, GPS-tracked travel routes, a simple rating system, and audio or written text narratives explaining each photo's content.

2. Discuss. Citizen scientists come together to review their data, identify main themes, and reach consensus around priorities for action.

3. Advocate. The study team or trained community facilitators support the citizen scientists in using their data to advocate for change, connecting them with relevant decision-makers and other stakeholders to whom they present their findings and recommendations.

4. Change. A period of collaborative action usually follows, in which changes to the environment are made.

\section{Participant Recruitment and Enrollment}

The study was advertised online (social media, websites, e-newsletters) and with flyers in high-traffic park areas. Eligible participants were St. Louis area residents 18 years or older who indicated visiting or traveling through the park at least once a week. Previous Our Voice studies have shown that citizen scientist consensus can be achieved with as few as 8-15 participants per user group and that attrition often occurs across study phases. Because of this, we set an enrollment cap of 15 participants per user group (one for recreational park users, and one comprising park commuters). Enrollment occurred on a rolling basis during August-November, 2020. Participants were compensated with $\$ 25$ gift cards for participation in each of the first three steps (Steps 1-3), for a potential total of $\$ 75$ in compensation.

\section{Adapting the Our Voice method during COVID-19}

COVID-19 stay-at-home orders were issued in St. Louis County and the City of St. Louis in late March, 2020 and remained active throughout the duration of this study. To effectively administer the Our Voice methodology while adhering to local guidelines and ensuring the safety of participants, the study team worked with Stanford Our Voice methods experts to modify study protocol. All contact with participants, including enrollment, was managed through email and phone; citizen scientists engaged in data collection alone (versus with a study team member), with remote guidance provided by study staff; meetings and training were facilitated virtually via videoconferencing software.

Following enrollment, each citizen scientist was contacted via email or phone to schedule a virtual training session on use of the Discovery Tool. In some Our Voice studies, study team members or trained community 
facilitators accompany citizen scientists on their data collection walks to guide them in using the Discovery Tool. To accommodate physical distancing recommendations, participants in this study received a 30-minute virtual training and were able to choose between engaging in discovery walks with a study team member (with physical distancing) or alone. All citizen scientists elected to go alone and use the Discovery Tool application on personal mobile devices. While discovery walks occurred at a time of each citizen scientist's choosing, they were scheduled to ensure that a study team member could serve on-call in case of any difficulty using the Discovery Tool.

The second and third steps of Our Voice constitute two sets of facilitated meetings in which citizen scientists engage in collaborative analysis of their data and present their findings to policymakers, respectively. While these meetings typically occur in person, implementation during COVID-19 involved a series of modifications. Each meeting was facilitated virtually using Zoom videoconferencing software. All meeting participants received agendas and other meeting materials in PDF documents via email several days prior to each meeting.

\section{Data Collection Procedures}

The study was approved by the Washington University in St. Louis Institutional Review Board (IRB ID\#: 202005157).

\section{Our Voice Step 1}

Citizen scientists trained on use of the Discovery Tool during August-November, 2020 and conducted discovery walks in August-December, 2020. Citizen scientists were offered a study-provided device for use of the Discovery Tool; however, all participating citizen scientists elected to use their personal mobile devices. Citizen scientists were informed that discovery walks could be performed during exercise, on bicycles, or by other active transport modes. Citizen scientists were asked to take their usual routes to and through the park, following their usual routine (exercise, active travel, etc.), and capture photographs and audio or text narratives of facilitators ("good" features) and barriers ("bad" features) to park use and access with the following prompt: What makes it easy or enjoyable to use the park? What hinders or prevents you from using the park? (see Appendix for Figure 2). Anonymous data collected using the Discovery Tool was uploaded to a password-protected online data portal on a secure server hosted by Stanford University. Each audio recording was automatically transcribed within the data portal platform, and transcripts were edited for accuracy by study staff.

\section{Data Cleaning and Preliminary Analysis}

To streamline the collaborative analytical process to be performed by citizen scientists at community meetings, the study team conducted a preliminary analysis of Discovery Tool data. First, we identified discovery walks as belonging to either the commuter or recreation user group. Second, each photo narrative was reviewed by at least two study team members in a thematic analysis. Third, each reviewer created a list of codes related to themes identified in photo narratives (e.g., "safety concerns," "park institutions," "maintenance") and performed a count of the number of times a theme was coded (data points could be coded as belonging to more than one theme). The study team met virtually to determine the 7-10 most common themes. During this step, the study team also removed "extra" discovery walks erroneously created with the Discovery Tool mobile application by citizen scientists.

\section{Our Voice Step 2}

Recreation and commuter user groups met for community meetings in December 2020 and January 2021, respectively. Prior to each meeting, discovery walk data from each user group was pooled and evenly split into five subsets for recreational users and three subsets for commuters. Data subsets were compiled in PDF documents containing 30-50 discovery walk photos accompanied by their text narratives, ratings, and maps showing the location of each photo. Citizen scientists were randomly assigned a subset of their user group's data, with 3-4 for each data subset. Citizen scientists were also provided with the study team's preliminary analysis, framed as a set of "suggested themes." Citizen scientists were asked to review these materials prior to the meeting and encouraged to make additions or edits to the list of suggested themes.

During the virtual meetings, citizen scientists were assigned to breakout rooms by data subset, with a study staff member available to assist if necessary. In these groups, citizen scientists were asked to reach agreement on 3-4 priorities for action (based on importance and feasibility for change) and draft potential solutions. After $30-40$ minutes of breakout room discussions, citizen scientists were reconvened. Each subgroup presented their priority themes and solution ideas. The Zoom polling feature was used to select the top three themes and associated solution ideas for which citizen scientists would advocate during the subsequent meeting between citizen scientists and park stakeholders (Step 3). Finally, each group selected two volunteer representatives, one for recreational users and one for commuters, to present their findings to stakeholders.

\section{Our Voice Step 3}

Prior to the citizen scientist-stakeholder meeting, the two volunteer representatives attended a virtual meeting with study staff, who shared strategies for effective communication of the research process, including data collection, analysis, and results.

The citizen scientist-stakeholder meeting occurred virtually in March 2021. Commuters and recreational park users presented their findings and solution ideas to park stakeholders, who responded to the issues raised and engaged in active discussion. Stakeholders were convened by the study team in collaboration with the nonprofit park 
conservancy, with whom many stakeholders had existing relationships and whose leadership also attended.

\section{Our Voice Step 4}

During the citizen scientist-stakeholder meeting, consensus was reached among a variety of park stakeholders on key issues requiring immediate and longerterm actions to improve park access and experience for park users. Members of the study team took field notes during the meeting, and subsequently conducted a thematic analysis to categorize those priorities and action items that received mutual verbal approval by citizen scientists and stakeholders.

\section{Results}

\section{Characteristics of Citizen Scientists}

Twenty-three citizen scientists participated in discovery walks ( 9 commuters and 14 recreational users). Of these, 20 attended their community meeting to analyze data and prioritize issues and solutions (Step 2), and 13 attended the citizen scientist-stakeholder meeting (Step 3).

Sociodemographic characteristics of the citizen scientists are shown in Table 1. Preliminary PARCS data analysis of direct observation and intercept survey data (data not shown) has found that park visitors are predominantly adults, white, and highly educated (having earned a 4-year college degree or more). In terms of race and education, the demographic composition of the citizen scientists largely reflect the makeup of frequent park users. However, while preliminary analysis indicates that park visitorship is wellbalanced by gender, the majority of Our Voice citizen scientists $(65.2 \%)$ were female.

Citizen scientists across both groups shared several characteristics. Most $(n=16,69.6 \%)$ lived in the City of St. Louis and accessed the park by walking ( $n=14,60.9 \%)$, suggesting residence close to the park. Similar proportions of recreation $(71.4 \%)$ and commuter $(77.8 \%)$ users reported visiting the park weekly, with 4 total daily park users $(17.4 \%)$. Most reported engaging in multiple activities during their park visits, with exercise $(82.6 \%)$, leisure walking $(56.5 \%)$, time in nature $(52.2 \%)$, and socializing with friends and family $(52.2 \%)$ being the most frequently reported activities. Recreational citizen scientists had a higher rate of personal vehicle ownership (100\%) than commuters $(66.7 \%)$ and were more likely to report membership in a civic group or community organization $(78.6 \%)$ than commuters $(22.2 \%)$.

Table 1. Sociodemographic and park visit related characteristics of Our Voice citizen scientists, August 2020-January 2021, (N= 23).

\begin{tabular}{|c|c|c|c|}
\hline \multirow[t]{2}{*}{ Characteristic } & $\begin{array}{c}\text { Total } \\
(\mathrm{N}=23)\end{array}$ & Commuter & $\begin{array}{c}\text { Recreation } \\
(\mathrm{n}=14)\end{array}$ \\
\hline & $\mathrm{n}(\%)$ & $\mathrm{n}(\%)$ & $\mathrm{n}(\%)$ \\
\hline \multicolumn{4}{|l|}{ Sociodemographic characteristics } \\
\hline \multicolumn{4}{|l|}{ Age } \\
\hline$<35$ years & $10(43.5)$ & $3(33.3)$ & $7(50)$ \\
\hline $35-64$ years & $10(43.5)$ & $6(66.7)$ & $4(28.6)$ \\
\hline$\geq 65$ years & $3(13)$ & 0 & $3(21.4)$ \\
\hline \multicolumn{4}{|l|}{ Sex } \\
\hline Male & $8(34.8)$ & $5(55.6)$ & $4(28.6)$ \\
\hline Female & $15(65.2)$ & $4(44.4)$ & $10(71.4)$ \\
\hline \multicolumn{4}{|l|}{ Race } \\
\hline White & $22(95.6)$ & $9(100)$ & $13(95.9)$ \\
\hline Black / African American & $1(4.3)$ & 0 & $1(7.1)$ \\
\hline \multicolumn{4}{|l|}{ Educational attainment } \\
\hline Less than college & $1(4.3)$ & 0 & $1(7.1)$ \\
\hline 4-year college degree & $9(39.1)$ & $2(22.2)$ & $7(50)$ \\
\hline Graduate training & $13(56.5)$ & $7(77.8)$ & $6(42.8)$ \\
\hline \multicolumn{4}{|l|}{ Yearly household income } \\
\hline$\$ 15,000-\$ 49,000$ & $6(26.1)$ & $3(33.3)$ & $3(21.4)$ \\
\hline$\$ 50,000-\$ 149,999$ & $11(47.8)$ & $4(44.4)$ & $7(50)$ \\
\hline$\geq \$ 150,000$ & $6(26.1)$ & $2(22.2)$ & $4(28.6)$ \\
\hline
\end{tabular}


Car ownership

$$
\text { Owns or leases a personal car }
$$

Does not own or lease a personal car

Place of residence

$$
\begin{array}{r}
\text { St Louis City (MO) } \\
\text { St Louis County (MO) } \\
\text { St Clair (IL) }
\end{array}
$$

Civic group or community organization membership ${ }^{1}$

$$
\begin{gathered}
\text { Yes } \\
\text { No }
\end{gathered}
$$

Park Use Frequency and Mode of Travel

Frequency of visits to the park

$$
\begin{array}{r}
\text { Monthly } \\
\text { Weekly } \\
\text { Daily } \\
\quad
\end{array}
$$

Most common mode of travel to the park

Walking
Bicycle
Public transit
Personal Car

Park-based activities

I use the park for exercising/working out

I actively commute (walk or bike) through the park

Note.

${ }^{1}$ Refers to participation or membership in the past 12 months on a neighborhood association, parent-teacher association, political organization, volunteer association, or advocacy group.

${ }^{2}$ Park facilities included museums, a visitor center, a theater, and a zoo, among other public facilities located within the park.

\section{Discovery Tool Data and Thematic Analysis}

\section{Our Voice Step 1 Results: Process Indicators}

During discovery walks, citizen scientists collected 373 unique data points (a photo with an accompanying rating and audio or text narrative) regarding facilitators and barriers to park access and use (mean data points per citizen scientist $=13.8 ; \mathrm{SD}=9.5)$. While all citizen scientists were asked to take only one discovery walk through the park, some elected to document more than one route to provide a comprehensive assessment of their usual park experience. After removal of non-valid data (e.g., a citizen scientist logging a "new walk" by error), citizen scientists logged a total of 27 discovery walks. 
Commuters conducted 10 discovery walks (mean duration $=31.8$ minutes, $\mathrm{SD}=20.2$ minutes), with one citizen scientist performing two walks to capture their regular use of distinct park areas. Recreation users engaged in 17 walks (mean duration $=43.4$ minutes, standard deviation $=26.9$ minutes), with two participants logging two or more walks. Nearly half of all data points $(n=179$, $48 \%$ ) reflected facilitators to park use and $137(36.7 \%)$ data points reflected barriers to park use. Fifty-seven (15.3\%) reflected both facilitators and barriers.

\section{Barriers and Opportunities for Park Access and Use: Thematic Analysis}

Preliminary thematic analysis by the study team revealed ten major themes within recreation citizen scientists' data, as follows: general problems with park access, underutilized green space, visitor adherence to pathway signage, appreciation for natural spaces, park safety, COVID-19 impacts, park museums and institutions as anchors for park visits, bathroom access, infrastructure maintenance issues, and the presence of multipurpose spaces for activity. Seven themes were captured within commuter data, as follows: appreciation for COVID-19 temporary road closures, issues with driver behavior, appreciation for natural spaces, need for more and better crosswalk signage, varying quality of crosswalks, varying quality of park entrances, and the presence of multipurpose spaces for activity.

\section{Our Voice Step 2 Results: Citizen Scientist Analysis of Barriers and Facilitators of Park Use}

The main themes generated by the study team's preliminary analysis were confirmed by each user group. Citizen scientists noted the frequent capture of data on park infrastructure, which they found was often insufficiently supportive of pedestrians and cyclists compared to motor vehicles. Relatedly, both groups cited concerns about injury by motor vehicles (e.g., speeding, cars rolling through stop signs, failure to yield at crosswalks and trail intersections) throughout the park. In addition, both groups independently discussed the need for more comprehensive signage throughout the park, particularly regarding information about facilities such as restroom access (recreation group), park entrances (commuter group), and user behavior on pedestrian-cyclist paths and trails (both groups). Finally, each group noted a subset of data points specifically regarding features of park use or visitor behavior unique to the COVID-19 pandemic (e.g., experiencing difficulty with physical distancing on paths or appreciating the road closures undertaken by the park to provide pedestrians and cyclists more space from each other).

\section{Prioritization of Themes and Solutions}

Despite the fact that each user group met separately, analysis during community meetings yielded five similar priority items across groups: (a) general park access issues, especially related to park entrances and streets; (b) appreciation for road closures; (c) traffic safety and driver behavior; (d) visitor use of shared pathways; and (e) infrastructure maintenance issues. A sixth theme, (f) restroom access, was uniquely identified by recreational citizen scientists.

In each group, citizen scientists elected to center their advocacy on issues of access and use of the park. The theme regarding general park access (a) was reframed to focus on enhancing park entrances and streets for pedestrians and cyclists rather than motorized vehicles - as one citizen scientist put it, "parks should be for people, not cars." Relatedly, citizen scientists praised the temporary road closures (b) throughout the park that had been prompted by COVID-19 physical distancing recommendations, and many expressed the desire to institute permanent road closures or a program of car-free days in some park areas after the physical distancing needs of the pandemic abated. Pedestrian and cyclist safety from motor vehicles (c) was characterized as an infrastructure issue, with calls for changes to crosswalk systems and street design that citizen scientists believed would be lowcost but high impact.

The path and trail infrastructure devoted to active transit was praised for its extensiveness and navigability.

However, citizen scientists unanimously agreed that use of trails was complicated (d) by user disregard for park conventions separating "heels" paths (for walkers and runners), which were often made of gravel or dirt, and paved "wheels" paths (for wheelchairs, strollers, and bicycles) — often leading to congestion and sometimes injury, and potentially making paths seem unwelcoming or inaccessible. Issues with path maintenance (e) such as cracked sidewalks, uneven paving, and insufficient water drainage were also prioritized as seemingly simple fixes.

Finally, the issue of unequal access to restrooms (f) throughout the park was presented as one of inclusion and equity. Citizen scientists observed that many freestanding park restrooms, particularly in areas without buildings or institutions, were locked. In community meetings, citizen scientists expressed confusion and frustration about this, indicating that the closure of restrooms without any signage detailing hours or availability made longer park visits inconvenient and gave the impression that visitors were not welcomed to stay.

\section{Our Voice Step 3 Results: Citizen Scientist Advocacy to Park Stakeholders}

As both groups independently prioritized most of the same main themes (a-e), and upon agreement of the commuter group to include improved restroom access (f) as another key priority, the recreation and commuter user groups were combined prior to the citizen scientiststakeholder meeting. The two volunteer representatives worked collaboratively to combine findings and align priorities. Through this collaboration between representatives and email correspondence with the other participating citizen scientists, they agreed to present the six priority themes and twelve particular solution ideas to park stakeholders, as seen in Table 2. 
Table 2. Analytic themes and associated solution ideas determined by commuter and recreation citizen scientists.

\begin{tabular}{|c|c|c|c|}
\hline Theme & Description & Representative Quote or Photo & Solutions Proposed \\
\hline
\end{tabular}

\begin{tabular}{ll}
\hline 2. Road closures & Park road closures \\
& prompted by COVID-19 \\
& physical distancing \\
& needs improved \\
& perceived pedestrian and \\
& cyclist safety and access
\end{tabular}

3. Traffic safety Significant automobile and driver presence in the park behavior yields safety concerns for pedestrians and cyclists not very welcoming."

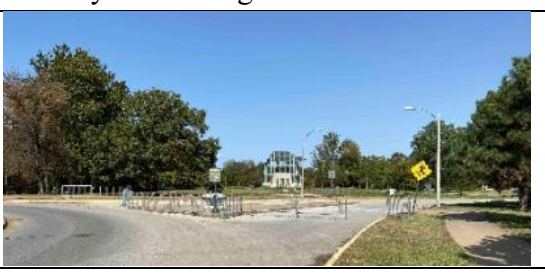

"This crosswalk is an absolute nightmare. Pedestrian/bike traffic is high (as it should be) but there are also lots of cars here. It's only stop signs and cars have almost hit me multiple times..."
- Institute permanent or more extensive road closures after the pandemic abates

\section{Visitor adherence to pathway signs}

\section{Existing designations of} separate paths for "heels" and "wheels" are not adhered to by visitors, which can impact user safety

\section{"I think the directional signage on the} lanes under the pavement here underneath this tunnel could use a little bit better signage; maybe be nice to separate cyclists and pedestrians altogether, divide them on either side, because as a cyclist you can come through here really fast...but it can scare the pedestrians..."

\begin{tabular}{ll}
\hline 5. Maintenance & Need for repairs to, or \\
and infrastructure & expansion of, pedestrian \\
quality & and cyclist infrastructure \\
& (e.g., crosswalks, \\
& sidewalks)
\end{tabular}

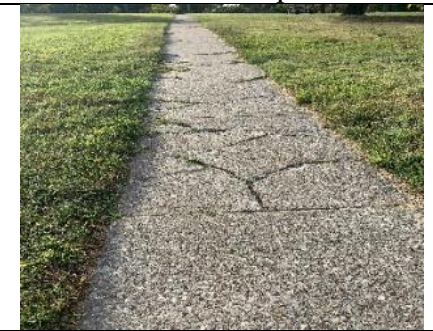

6. Restroom "These bathrooms are almost always access accessible restrooms throughout park outside of institutions (e.g., museums) or office buildings locked or are closed. They should be open any time the park is open. And they should add more restrooms to the park."
- $\quad$ Install stop/yield signs on paths at all road crossings; improve crosswalk markings

- Install crosswalks at sidewalk height with curb extensions or pavers to decrease vehicle speed

- Decrease turning radii at vehicle intersections

- $\quad$ Decrease posted speeds within the park to 15-20 miles per hour

- $\quad$ Paint heels/wheels signage on paved paths

- $\quad$ Provide guidance on path 'rules' in pathway signage, at park visitor center, and on maps or marketing materials

- Create annual maintenance schedule for paths and trails
Invited stakeholders were selected given the six priority themes and included: the local public transit authority; the city Office of Planning and Urban Design; the city
Department of Parks, Recreation, and Forestry; the park nonprofit conservancy; and the park advisory board. Citizen scientist representatives defined the meeting agenda 
with the study team. In the first part of the meeting, the study team facilitated introductions and the two citizen scientist volunteers gave a brief overview of the Our Voice methodology. Subsequently, citizen scientist volunteers reported their findings. They began by reporting commonly cited positive features of the park, such as its natural beauty and variety of amenities; they then introduced the priority six themes, which were presented as barriers to use or areas for improvement and were accompanied with proposals for solutions.

\section{Our Voice Step 4: Immediate Outcomes of the Our Voice Process}

Following the citizen scientists' presentation, stakeholders expressed gratitude for receiving communitygenerated data and feedback on park use and access. They indicated that the citizen scientists' analysis reinforced existing master plan guidelines and recommendations from other studies recently undertaken about park access and internal navigation. Stakeholders found that the data produced by citizen scientists validated design practices and scheduled park changes, and provided new guidance for prioritizing capital improvement and maintenance projects. Leaders from the nonprofit park conservancy expressed interest in creating a process for continuing community feedback sessions.

Similarly, many citizen scientists expressed gratitude and appreciation for being a part of the Our Voice process. Citizen scientists gained information about park operations and avenues for continued engagement with park decisionmaking, enhancing their understanding and engagement with a park they frequent. One citizen scientist reflected on their use of the Discovery Tool and their training in thematic analysis, saying, "I've never been called a scientist before."

Thematic analysis of the notes taken by the study team during the citizen scientist-stakeholder meeting yielded seven action items presented to the nonprofit park conservancy. Short-term actions, which could be taken unilaterally by the park conservancy or the city Department of Parks and Recreation, included (a) posting restroom access information on maps and facilities; (b) sharing more widely an online park map providing restroom and pathway information; and (c) instituting temporary signage campaigns regarding appropriate path use in high-traffic areas. Medium-term actions included (d) establishing regular community feedback meetings with park users and neighbors and (e) considering planned street closures following the COVID-19 pandemic. Long-term actions focused on alignment with existing strategies for park design and access, including (f) collaborating with regional transit authorities to reduce car dependency in the park and (g) incorporating citizen scientists' priorities and solution ideas within the park master plan. All stakeholders demonstrated interest in finding ways to implement these suggested actions, with particular interest in improving park signage regarding path and trail use and restroom access. Follow-up at the completion of the PARCS Our Voice study will track progress on these action items.

\section{Discussion}

Our study of frequent recreational and commuter users of a metropolitan park in St. Louis demonstrated that Our Voice is a feasible and effective method for assessing drivers of access and use of large metropolitan parks. The similarity in findings across seemingly different user groups (recreational users and commuters) reinforces the richness of the Our Voice method, in which a small number of citizen scientists from a given community (in this case, frequent park users) can provide sufficient information about that community's overall experience, needs, and priorities (Rosas et al., 2016; Rubio et al., 2021). This makes Our Voice a powerful method for assessing largescale community amenities like major metropolitan parks, which serve millions of people per year and whose service areas are geographically large and demographically diverse. This is especially true when contrasting Our Voice with more traditional quantitative approaches (e.g., a representative survey of park users), which require complex sampling schemes and large samples to ensure internal and external validity (Fermino et al., 2015; Schmidt et al., 2019).

In contrast to findings from previous Our Voice studies in which different community user groups (e.g., different schools or age groups) identified different priority themes, the commuter and recreation user groups in this study largely prioritized the same issues (Rodriguez et al., 2019; Rosas et al., 2016). The only exception was the theme of restroom access, which was initially prioritized by recreational citizen scientists-likely due to their use of the park as a destination as opposed to a route to get to workuntil the two groups merged, and commuters agreed that it was an important issue for advocacy. This implies that adults who are frequent users of metropolitan parks (regardless of their main reason for park use) have more commonalities than differences.

Engaging in negotiation and having to reach agreement on priority themes allowed citizen scientists to learn about the challenges faced by other types of park users and encouraged collaboration. Our results suggest that participation in the Our Voice process may yield additional benefits for users and communities beyond the main goal of assessing and improving park access and use. These cobenefits could include increased empathy, social cohesion, and awareness of the community's broader needs. This finding is consistent with those of previous Our Voice studies, which have reported positive changes in some of these co-benefits (King, King, et al., 2020; King, OdunitanWayas, et al., 2021). Future Our Voice studies should expand their examination of these important outcomes.

This study also demonstrated the flexibility of the Our Voice method in adapting to unplanned changes in the research setting or to the results of citizen scientist analysis. Reviewing citizen scientists' priorities following user group 
community meetings made it clear that the advocacy process would be more efficient and effective if the two user groups could reach consensus on common needs and advocate together. The study team was able to easily pivot the study to accommodate this, and citizen scientists unanimously approved the change. Similarly, protocol changes implemented during the COVID-19 crisis had positive implications for study cost, reach, and scalability. Offering virtual gatherings eliminates travel and room rental costs; it may also increase attendance and participation by citizen scientists and community stakeholders, who can participate regardless of their location and use videoconferencing features (e.g. chat boxes or emoji reactions) to communicate in whatever way is most comfortable. With appropriate facilitation and planning, studies focusing on citizen science or CBPR are not necessarily limited to in-person gatherings.

For both citizen scientists and stakeholders, Our Voice presented an effective method for bridging the gaps between users of metropolitan parks and those who plan and maintain them. PARCS Our Voice enabled bottom-up policymaking by equipping frequent park visitors with the resources and information needed to address barriers to park use - their own, as well as others'. For park stakeholders, engagement in this process demonstrated a viable method of enshrining community input in future planning - a hopeful sign of continued citizen participation in decision-making regarding a critical regional health asset.

This study marks a unique, remotely-facilitated application of the Our Voice approach to evaluating metropolitan park access and use. A number of Our Voice studies have assessed parks as a part of a wider set of neighborhood features rather than as unique environments in and of themselves (Buman, Winter, et al., 2013; Rosas et al., 2016; Winter, Goldman, et al., 2016). Studies that evaluated parks as their main focus have centered on parkbased programs and used citizen scientist feedback to help characterize use of a small, temporary pop-up park (Rubio et al., 2021; Winter, Sheats, et al., 2020). The geographic scale and broad scope of assessment in our study provided a novel test of the applicability of Our Voice to more complex park environments. For example, this study enjoyed a wider range and higher rate of stakeholder participation compared to previous park-based Our Voice studies - in part because the multi-stakeholder governance of this major metropolitan park demanded it. This demonstration of the Our Voice method offers important lessons for other cities seeking to identify community priorities and determine enhancements, activities, or programming that would improve access to and utilization of metropolitan parks.

Table 3 shows a list of recommended practices for using the Our Voice method to identify barriers and opportunities for access and use of metropolitan parks or other similar urban hubs and assets. Ensuring that citizen scientists are reasonably representative of the community under investigation is critical. In this case, while citizen scientists were not demographically representative of the greater St. Louis region, as a group, they did generally resemble the make-up of usual park visitors per preliminary PARCS analysis. We also recommend examining all possible uses of the park or asset to determine which particular user groups may offer unique perspectives on use and access, and then capitalizing on any existing relationships to share recruitment materials widely within these communities. Once enrolled, citizen scientists ought to receive clear instruction on data collection and analysis, particularly if there are multiple amenities or uses under observation. Citizen scientist priorities should determine the course of advocacy activities, including the stakeholder organizations invited to the advocacy meeting. Given the complex governance of large metropolitan assets, and in order to streamline advocacy discussions, meeting invitations should focus on individuals with real decision-making power in the areas of most concern to the citizen scientists. Finally, we recommend embracing the use of technology such as videoconferencing software to offer citizen scientists choices in how they engage in data collection, analysis, and advocacy - so long as all participants have equal access to study information, activities, and those communication technologies. Levels of technology access (i.e., smartphone ownership, internet access, etc.) and technological literacy among the target population should be assessed when deciding to offer these types of options for citizen scientist engagement (Pratt et al., 2012; Salvo et al., 2014; Wallis et al., 2017). This point notwithstanding, in an ongoing cluster-randomized trial of Our Voice in 10 affordable senior public housing sites in two northern California counties, the majority of community residents with low technology literacy at the start of the trial were able to learn how to successfully use similar communication technologies with the help of family members, other residents, and study staff. 
Table 3. Recommended practices for the use of Our Voice to promote equity in access to large parks and other major metropolitan hubs or assets

\begin{tabular}{|c|c|}
\hline Topics & Recommendations \\
\hline $\begin{array}{l}\text { Recruitment } \\
\text { and community } \\
\text { representation }\end{array}$ & $\begin{array}{l}\text { - Assess the park or asset's range of possible activities and amenities to determine different user groups } \\
\text { - Use demographic information regarding park/asset user base or geographic service area to determine } \\
\text { catchment area or target groups for participation } \\
\text { - Aim to enroll 8-15 citizen scientists per user group to achieve saturation } \\
\text { - Capitalize on existing relationships between park/asset and community organizations, institutions, or } \\
\text { businesses to facilitate recruitment } \\
\text { Prepare to potentially aggregate user groups if citizen scientist analysis reveals predominantly common } \\
\text { main themes and similar priorities for change }\end{array}$ \\
\hline $\begin{array}{l}\text { Data collection } \\
\text { (discovery } \\
\text { walks) and } \\
\text { analysis }\end{array}$ & 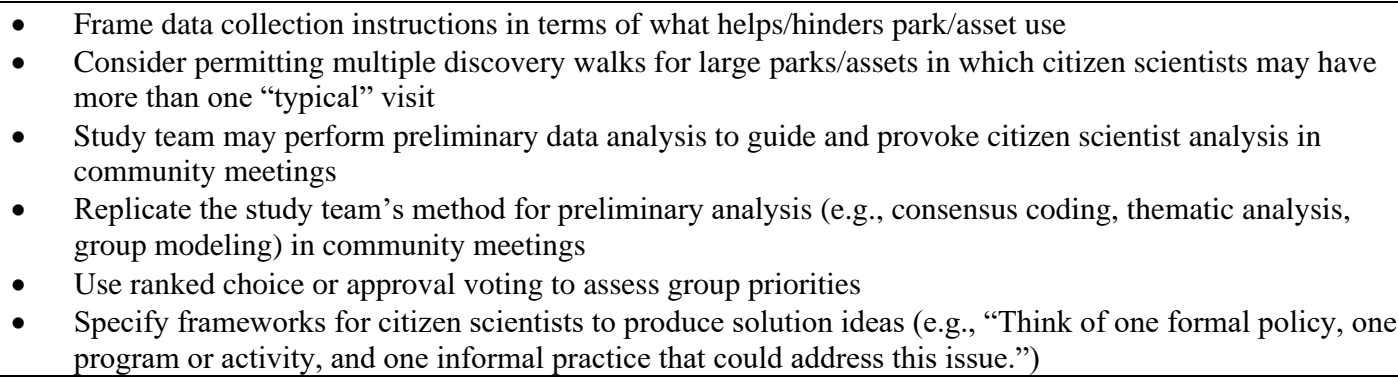 \\
\hline $\begin{array}{c}\text { Stakeholder } \\
\text { engagement and } \\
\text { citizen scientist } \\
\text { advocacy }\end{array}$ & $\begin{array}{l}\text { - Invite stakeholders based on main themes/priorities for advocacy as well as local governance (e.g., } \\
\text { infrastructure issues may be addressed by city or state departments of transportation as well as park/asset } \\
\text { operators) - but focus invitations on individuals with decision-making power } \\
\text { Capitalize on existing relationships between park/asset operators and stakeholder organizations to invite } \\
\text { stakeholders to participate } \\
\text { Produce a "meeting guide" for stakeholders including a brief explanation of the Our Voice process, } \\
\text { descriptions of citizen scientist data (e.g., number of photos, frequency of facilitators vs. barriers to } \\
\text { access), and the results of study team preliminary analysis } \\
\text { Consider not providing stakeholders with advance notice of solution ideas or other citizen scientist "asks" } \\
\text { so as not to bias stakeholder responses } \\
\text { Produce and share an advance meeting agenda clearly outlining expectations for participation (e.g., } \\
\text { stakeholder responses to citizen scientist priorities; open discussion/Q\&A session; etc.) }\end{array}$ \\
\hline $\begin{array}{l}\text { Adapting study } \\
\text { protocols to } \\
\text { virtual } \\
\text { participation }\end{array}$ & 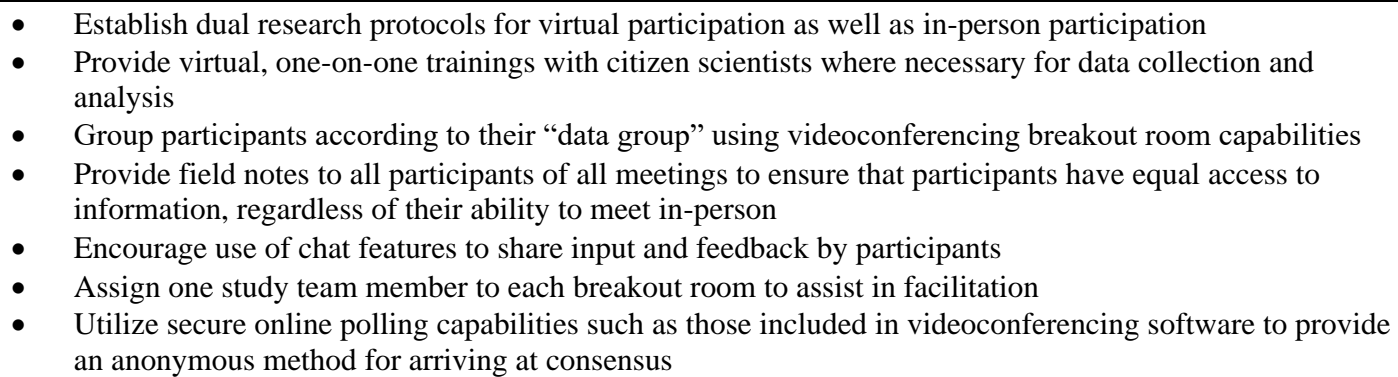 \\
\hline
\end{tabular}

Finally, beyond the study of metropolitan parks, COVID-19-prompted lessons in the adaptability of Our Voice protocols that can be generalized to any study situation in which in-person interactions between researchers, community members, and stakeholders is challenging or impracticable. The increased capacity for remote participation has significant positive implications for inclusion, cost, reach, and scale-up in future Our Voice projects.

\section{Limitations}

These findings must be considered in light of the study limitations. The citizen scientists recruited for this phase of the PARCS Our Voice study were self-identified frequent park users who would be more likely to have positive opinions of the park, and less likely to encounter meaningful obstacles to accessing or using it frequently. In addition, citizen scientists were more likely to be female than the general visitor population of the park. There is further potential for selection bias in the use of the park conservancy's e-newsletter and social media accounts for recruitment, as their readership is more likely to be engaged with park operations than other frequent users. As mentioned in the results, the study sample in this first phase was mostly white and college-educated-unrepresentative of the regional St. Louis population and the populations of many neighborhoods close to the park, but representative of frequent (weekly or more) park users according to preliminary analysis of quantitative, direct observation data 
from the parent PARCS study. Park-proximate communities with low rates of park use will be the focus of the second phase of PARCS Our Voice, and part of an indepth, mixed-methods study of low-utilization areas that will identify park access and perceptions of non-users. Analysis of zip code and visit frequency data, collected over several months in intercept surveys across the park in the first phase of the study, enabled the identification of these target neighborhoods; thus, sequencing Our Voice phases by these citizen scientist groups was necessary.

The participant pool underwent some attrition as study phases progressed. Though 26 citizen scientists enrolled in the study, three left the study prior to performing discovery walks. Of the 23 remaining, 20 attended their respective community meetings, and 13 attended the citizen scientiststakeholder meeting. Similar levels of attrition have been noted in other Our Voice studies, given that residents typically vary in their comfort levels concerning discussions with local decision-makers in particular (Rubio et al., 2021; Winter, Goldman, et al., 2016). As in those studies, PARCS citizen scientists were able to come to consensus on analysis and priorities regardless of attrition. While issues of attrition and selection bias are of central importance in research aimed at individual-level outcomes, in research such as this where the primary outcomes of interest are at higher levels of impact (i.e., changes in local environments and policies), such issues become less critical (King, Odunitan-Wayas, et al., 2021). Regardless, finding methods to expand participation across the full "by the people" citizen science process is recommended as a means for further enriching the process for residents.

Although the citizen scientist-stakeholder meeting enjoyed high participation rates by invited stakeholder representatives, the processes of follow-up on the action items from that meeting have not yet been fully defined. This is partly because the PARCS Our Voice project will since undergo a second phase in which non-park users also collect and analyze data and generate recommendations. Data from both phases will be synthesized to develop overall recommendations for enhancing equity in access and use of the park. Finally, like all study methods, Our Voice works best when used in concert with other methodologies as part of a comprehensive mixed-methods study. When complete, PARCS will integrate the results of the Our Voice analysis with additional evaluations, including standardized SOPARC observations, intercept surveys, stakeholder interviews, and focus groupsallowing a fuller understanding of these results and their implications (McKenzie et al., 2016).

\section{Correspondence should be addressed to}

Deborah Salvo, $\mathrm{PhD}$

Brown School at Washington University in St. Louis

Campus Box 1196, One Brookings Drive
St. Louis, MO 63130, USA

dsalvo@wustl.edu

314-935-4131

Aine O'Connor 0000-0002-8903-4092

Abby C. King: 0000-0002-7949-8811

Ann Banchoff: 0000-0002-0185-2200

Amy Eyler: 0000-0001-8417-1656

Rodrigo Reis: 0000-0002-9872-9865

Ross C. Brownson: 0000-0003-4260-2205

Eugen Resendiz: 0000-0002-4500-879X

Derek Holland: 0000-0002-0134-2673

Deborah Salvo: 0000-0002-9726-0882

\section{Acknowledgements}

Our earnest gratitude to PARCS Our Voice citizen scientists and to park stakeholders, without whom this study would not have been possible. Thank you to Isela Blanco-Velazquez, from Stanford University, for mentorship on facilitating Our Voice community meetings, and to Irvin Szeto, from Stanford University, for technical assistance with the Discovery Tool data portal. We also appreciate the assistance of William Effah, MD, and Fatemeh Naghiloo, MPH, MA, of Washington University in St. Louis with facilitation, thematic coding, and notetaking. Data collection was in part supported by Forest Park Forever (FPF), a non-profit agency. This work was also supported by the Prevention Research Center in St. Louis (CDC \#U48DP006395). The content of the publication is solely the responsibility of the authors and does not necessarily represent the views of FPF or of the CDC.

\section{Author Contributions}

Conceptualization, D.S.; Data curation, E.R.; Formal analysis, A.O.C., E.R., and D.H.; Funding Acquisition, D.S., R.R., A.E., and R.B.; Investigation, A.O.C. and D.S.; Methodology, D.S., A.K., A.B., D.H., and A.O.C.; Project Administration, D.H. and A.O.C.; Resources, A.B. and A.K.; Supervision, D.S.; Visualization, A.O.C. and E.R.; Writing - Original Draft, A.O.C. and D.S.; Writing Review \& Editing, D.S., A.O.C., A.B., A.E., A.K., E.R., R.B., and R.R.

\section{Conflict of interest statement:}

We have no conflicts of interest to disclose.

\section{Creative Commons License:}

This work is licensed under a Creative Commons Attribution-Noncommercial 4.0 International License (CC BY-NC 4.0). 


\section{References}

Bedimo-Rung, A. L., Mowen, A. J., \& Cohen, D. A. (2005). The significance of parks to physical activity and public health: A conceptual model. American Journal of Preventive Medicine, 28(2 SUPPL. 2), 159-168.

https://doi.org/10.1016/j.amepre.2004.10.024

Buman, M. P., Bertmann, F., Hekler, E. B., Winter, S. J., Sheats, J. L., King, A. C., \& Wharton, C. M. (2015). A qualitative study of shopper experiences at an urban farmers' market using the Stanford Healthy Neighborhood Discovery Tool. Public Health Nutrition, 18(6), 994-1000. https://doi.org/10.1017/S136898001400127X

Buman, M. P., Winter, S. J., Sheats, J. L., Hekler, E. B., Otten, J. J., Grieco, L. A., \& King, A. C. (2013). The stanford healthy neighborhood discovery tool: A computerized tool to assess active living environments. American Journal of Preventive Medicine, 44(4), e41. https://doi.org/10.1016/j.amepre.2012.11.028

Chrisinger, B. W., Ramos, A., Shaykis, F., Martinez, T., Banchoff, A. W., Winter, S. J., \& King, A. C. (2018). Leveraging citizen science for healthier food environments: A pilot study to evaluate corner stores in camden, New Jersey. Frontiers in Public Health, 6(MAR). https://doi.org/10.3389/fpubh.2018.00089

Doolittle, A. \& Faul, A. C. (2013). Civic engagement scale: A validation study. SAGE Open, 3(3), 1-7. https://doi.org/10.1177/2158244013495542

Ferdinand, A. O., Sen, B., Rahurkar, S., Engler, S., \& Menachemi, N. (2012). The relationship between built environments and physical activity: A systematic review. In American Journal of Public Health (Vol. 102, Issue 10, p. e7). American Public Health Association. https://doi.org/10.2105/AJPH.2012.300740

Fermino, R., Reis, R., Hallal, P. C., \& Kaczynski, A. T. (2015). Who are the users of urban parks? A study with adults from Curitiba, Brazil. Journal of Physical Activity and Health, 12(1), 58-67. https://doi.org/10.1123/jpah.2012-0482

Gascon, M., Mas, M. T., Martínez, D., Dadvand, P., Forns, J., Plasència, A., \& Nieuwenhuijsen, M. J. (2015). Mental health benefits of long-term exposure to residential green and blue spaces: A systematic review. In International Journal of Environmental Research and Public Health (Vol. 12, Issue 4, pp. 4354-4379). MDPI AG. https://doi.org/10.3390/ijerph120404354

Gelormino, E., Melis, G., Marietta, C., \& Costa, G. (2015). From built environment to health inequalities: An explanatory framework based on evidence. In Preventive Medicine Reports (Vol. 2, pp. 737-745). Elsevier Inc. https://doi.org/10.1016/j.pmedr.2015.08.019

González, S. A., Rubio, M. A., Triana, C. A., King, A. C., Banchoff, A. W., \& Sarmiento, O. L. (2021). Building healthy schools through technology-enabled citizen science: The case of the our voice participatory action model in schools from Bogotá, Colombia. Global Public Health. https://doi.org/10.1080/17441692.2020.1869285 
Humpel, N., Owen, N., \& Leslie, E. (2002). Environmental factors associated with adults' participation in physical activity. A review. In American Journal of Preventive Medicine (Vol. 22, Issue 3, pp. 188-199). Elsevier Inc. https://doi.org/10.1016/S0749-3797(01)00426-3

Israel, B. A., Coombe, C. M., Cheezum, R. R., Schulz, A. J., McGranaghan, R. J., Lichtenstein, R., Reyes, A. G., Clement, J., \& Burris, A. (2010). Community-based participatory research: A capacity-building approach for policy advocacy aimed at eliminating health disparities. American Journal of Public Health, 100(11), 2094-2102. https://doi.org/10.2105/AJPH.2009.170506

Israel, B. A., Schulz, A. J., Parker, E. A., \& Becker, A. B. (1998). Review of community-based research: Assessing partnership approaches to improve public health. In Annual Review of Public Health (Vol. 19, pp. 173-202). Annual Reviews 4139 El Camino Way, P.O. Box 10139, Palo Alto, CA 94303-0139, USA. https://doi.org/10.1146/annurev.publhealth.19.1.173 Jennings, V., Larson, L., \& Yun, J. (2016). Advancing sustainability through urban green space: Cultural ecosystem services, equity, and social determinants of health. International Journal of Environmental Research and Public Health, 13(2). https://doi.org/10.3390/ijerph13020196

Jimenez, M. P., DeVille, N. V., Elliott, E. G., Schiff, J. E., Wilt, G. E., Hart, J. E., \& James, P. (2021). Associations between Nature Exposure and Health: A Review of the Evidence. International Journal of Environmental Research and Public Health, 18(9), 4790. https://doi.org/10.3390/ijerph18094790

Kaczynski, A. T. \& Henderson, K. A. (2007). Environmental correlates of physical activity: A review of evidence about parks and recreation. In Leisure Sciences (Vol. 29, Issue 4, pp. 315-354). https://doi.org/10.1080/01490400701394865

Kaźmierczak, A. (2013). The contribution of local parks to neighbourhood social ties. Landscape and Urban Planning, 109(1), 31-44. https://doi.org/10.1016/j.landurbplan.2012.05.007

King, A. C., King, D. K., Banchoff, A., Solomonov, S., Natan, O. B., Hua, J., Gardiner, P., Rosas, L. G., Espinosa, P. R., Winter, S. J., Sheats, J., Salvo, D., Aguilar-Farias, N., Stathi, A., Hino, A. A., \& Porter, M. M. (2020). Employing participatory citizen science methods to promote age-friendly environments worldwide. International Journal of Environmental Research and Public Health, 17(5), 1541. https://doi.org/10.3390/ijerph17051541

King, A. C., Odunitan-Wayas, F. A., Chaudhury, M., Rubio, M. A., Baiocchi, M., Kolbe-Alexander, T., Montes, F., Banchoff, A., Sarmiento, O. L., Bälter, K., Hinckson, E., Chastin, S., Lambert, E. V., González, S. A., Guerra, A. M., Gelius, P., Zha, C., Sarabu, C., Kakar, P. A., ... Gardiner, P. A. (2021). Community-based approaches to reducing health inequities and fostering environmental justice through global youth-engaged citizen science. International Journal of Environmental Research and Public Health, 18(3), 1-29. https://doi.org/10.3390/ijerph18030892

King, A. C., Winter, S. J., Chrisinger, B. W., Hua, J., \& Banchoff, A. W. (2019). Maximizing the promise of citizen science to advance health and prevent disease. In Preventive Medicine (Vol. 119, pp. 44-47). Academic Press Inc. 
https://doi.org/10.1016/j.ypmed.2018.12.016

King, A. C., Winter, S. J., Sheats, J. L., Rosas, L. G., Buman, M. P., Salvo, D., Rodriguez, N. M., Seguin, R. A., Moran, M., Garber, R., Broderick, B., Zieff, S. G., Sarmiento, O. L., Gonzalez, S. A., Banchoff, A., \& Dommarco, J. R. (2016). Leveraging Citizen Science and Information Technology for Population Physical Activity Promotion. Translational Journal of the American College of Sports Medicine, 1(4), 30-44. https://doi.org/10.1249/tjx.0000000000000003

Kruizse, H., van der Vliet, N., Staatsen, B., Bell, R., Chiabai, A., Muiños, G., Higgins, S., Quiroga, S., Martinez-Juarez, P., Yngwe, M. A., Tsichlas, F., Karnaki, P., Lima, M. L., de Jalón, S. G., Khan, M., Morris, G., \& Stegeman, I. (2019). Urban green space: creating a triple win for environmental sustainability, health, and health equity through behavior change. In International Journal of Environmental Research and Public Health (Vol. 16, Issue 22). MDPI AG. https://doi.org/10.3390/ijerph16224403

Lee, R. E., Booth, K. M., Reese-Smith, J. Y., Regan, G., \& Howard, H. H. (2005). The Physical Activity Resource Assessment (PARA) instrument: Evaluating features, amenities and incivilities of physical activity resources in urban neighborhoods. International Journal of Behavioral Nutrition and Physical Activity, 2(1), 1-9. https://doi.org/10.1186/1479-5868-2-13

Leyden, K. M. (2003). Social Capital and the Built Environment: The Importance of Walkable Neighborhoods. American Journal of Public Health, 93(9), 1546-1551. https://doi.org/10.2105/AJPH.93.9.1546

Maas, J., van Dillen, S. M. E., Verheij, R. A., \& Groenewegen, P. P. (2009). Social contacts as a possible mechanism behind the relation between green space and health. Health and Place, 15(2), 586-595. https://doi.org/10.1016/j.healthplace.2008.09.006

McCormack, G. R., Rock, M., Toohey, A. M., \& Hignell, D. (2010). Characteristics of urban parks associated with park use and physical activity: A review of qualitative research. Health and Place, 16(4), 712-726. https://doi.org/10.1016/j.healthplace.2010.03.003

McKenzie, T. L., Cohen, D. A., Sehgal, A., Williamson, S., \& Golinelli, D. (2016). System for Observing Play and Recreation in Communities (SOPARC): Reliability and Feasibility Measures. Journal of Physical Activity and Health, 3(s1), S208S222. https://doi.org/10.1123/jpah.3.s1.s208

Moran, M. R., Werner, P., Doron, I., HaGani, N., Benvenisti, Y., King, A. C., Winter, S. J., Sheats, J. L., Garber, R., Motro, H., \& Ergon, S. (2017). Exploring the objective and perceived environmental attributes of older adults' neighborhood walking routes: A mixed methods analysis. Journal of Aging and Physical Activity, 25(3), 420-431. https://doi.org/10.1123/japa.2016-0165

Morgan Hughey, S., Kaczynski, A. T., Child, S., Moore, J. B., Porter, D., \& Hibbert, J. (2017). Green and lean: Is neighborhood park and playground availability associated with youth obesity? Variations by gender, socioeconomic status, and race/ethnicity. Preventive Medicine, 95, S101-S108. https://doi.org/10.1016/j.ypmed.2016.11.024 
Paulos, E., Honicky, R., \& Hooker, B. (2008). Citizen Science: Enabling Participatory Urbanism. In Handbook of Research on Urban Informatics (pp. 414-436). IGI Global. https://doi.org/10.4018/978-1-60566-152-0.ch028

Pratt, M., Sarmiento, O. L., Montes, F., Ogilvie, D., Marcus, B. H., Perez, L. G., Brownson, R. C., Alkandari, J. R., Andersen, L. B., Bauman, A. E., Blair, S. N., Bull, F. C., Craig, C. L., Ekelund, U., Goenka, S., Guthold, R., Hallal, P. C., Haskell, W. L., Heath, G. W., ... Wells, J. C. (2012). The implications of megatrends in information and communication technology and transportation for changes in global physical activity. In The Lancet (Vol. 380, Issue 9838, pp. 282-293). Elsevier B.V. https://doi.org/10.1016/S0140-6736(12)60736-3

Rodriguez, N. M., Arce, A., Kawaguchi, A., Hua, J., Broderick, B., Winter, S. J., \& King, A. C. (2019). Enhancing safe routes to school programs through community-engaged citizen science: Two pilot investigations in lower density areas of Santa Clara County, California, USA. BMC Public Health, 19(1), 1-11. https://doi.org/10.1186/s12889-019-6563-1

Rosas, L. G., Salvo, D., Winter, S. J., Cortes, D., Rivera, J., Rodriguez, N. M., \& King, A. C. (2016). Harnessing Technology and Citizen Science to Support Neighborhoods that Promote Active Living in Mexico. Journal of Urban Health, 93(6), 953973. https://doi.org/10.1007/s11524-016-0081-6

Rubio, M. A., Triana, C., King, A. C., Rosas, L. G., Banchoff, A. W., Rubiano, O., Chrisinger, B. W., \& Sarmiento, O. L. (2021). Engaging citizen scientists to build healthy park environments in Colombia. Health Promotion International, 36(1), 223234. https://doi.org/10.1093/heapro/daaa031

Rugel, E. J., Carpiano, R. M., Henderson, S. B., \& Brauer, M. (2019). Exposure to natural space, sense of community belonging, and adverse mental health outcomes across an urban region. Environmental Research, 171, 365-377. https://doi.org/10.1016/j.envres.2019.01.034

Saelens, B. E., Frank, L. D., Auffrey, C., Whitaker, R. C., Burdette, H. L., \& Colabianchi, N. (2006). Measuring Physical Environments of Parks and Playgrounds: EAPRS Instrument Development and Inter-Rater Reliability.

Sallis, J. F., Cerin, E., Conway, T. L., Adams, M. A., Frank, L. D., Pratt, M., Salvo, D., Schipperijn, J., Smith, G., Cain, K. L., Davey, R., Kerr, J., Lai, P. C., Mitáš, J., Reis, R., Sarmiento, O. L., Schofield, G., Troelsen, J., Van Dyck, D., ... Owen, N. (2016). Physical activity in relation to urban environments in 14 cities worldwide: A cross-sectional study. The Lancet, 387(10034), 2207-2217. https://doi.org/10.1016/S0140-6736(15)01284-2

Salvo, D., Banda, J. A., Sheats, J. L., Winter, S. J., Lopes dos Santos, D., \& King, A. C. (2017). Impacts of a Temporary Urban Pop-Up Park on Physical Activity and Other Individual- and Community-Level Outcomes. Journal of Urban Health, 94(4), 470-481. https://doi.org/10.1007/s11524-017-0167-9

Salvo, D., Reis, R. S., Sarmiento, O. L., \& Pratt, M. (2014). Overcoming the challenges of conducting physical activity and built environment research in Latin America: IPEN Latin America. Preventive Medicine, 69(S), S86-S92. https://doi.org/10.1016/j.ypmed.2014.10.014 
Schipperijn, J., Cerin, E., Adams, M. A., Reis, R., Smith, G., Cain, K., Christiansen, L. B., van Dyck, D., Gidlow, C., Frank, L. D., Mitáš, J., Pratt, M., Salvo, D., Schofield, G., \& Sallis, J. F. (2017). Access to parks and physical activity: An eight country comparison. Urban Forestry and Urban Greening, 27, 253-263. https://doi.org/10.1016/j.ufug.2017.08.010

Schmidt, T., Kerr, J., \& Schipperijn, J. (2019). Associations between neighborhood open space features and walking and social interaction in older adults-a mixed methods study. Geriatrics (Switzerland), 4(3). https://doi.org/10.3390/geriatrics4030041

Sheats, J. L., Winter, S. J., Romero, P. P., \& King, A. C. (2017). FEAST: Empowering Community Residents to Use Technology to Assess and Advocate for Healthy Food Environments. Journal of Urban Health, 94(2), 180-189. https://doi.org/10.1007/s11524-017-0141-6

Statistics Canada. (2013). General Social Survey: An Overview. www.statcan.gc.ca,

Tuckett, A. G., Banchoff, A. W., Winter, S. J., \& King, A. C. (2018). The built environment and older adults: A literature review and an applied approach to engaging older adults in built environment improvements for health. International Journal of Older People Nursing, 13(1). https://doi.org/10.1111/opn.12171

Tuckett, A. G., Freeman, A., Hetherington, S., Gardiner, P. A., \& King, A. C. (2018). Older adults using our voice citizen science to create change in their neighborhood environment. International Journal of Environmental Research and Public Health, 15(12). https://doi.org/10.3390/ijerph15122685

Vanaken, G. J., \& Danckaerts, M. (2018). Impact of green space exposure on children's and adolescents' mental health: A systematic review. In International Journal of Environmental Research and Public Health (Vol. 15, Issue 12). MDPI AG. https://doi.org/10.3390/ijerph15122668

Wallis, L., Blessing, P., Dalwai, M., \& Shin, S. Do. (2017). Integrating mHealth at point of care in low- and middle-income settings: The system perspective. Global Health Action, 10(sup3). https://doi.org/10.1080/16549716.2017.1327686

Winter, S. J., Goldman Rosas, L., Padilla Romero, P., Sheats, J. L., Buman, M. P., Baker, C., \& King, A. C. (2016). Using Citizen Scientists to Gather, Analyze, and Disseminate Information About Neighborhood Features That Affect Active Living. Journal of Immigrant and Minority Health, 18(5), 1126-1138. https://doi.org/10.1007/s10903-015-0241-x

Winter, S. J., Sheats, J. L., Salvo, D., Banda, J. A., Quinn, J., Rivera, B. R., \& King, A. C. (2020). A Mixed Method Study to Inform the Implementation and Expansion of Pop-Up Parks for Economic, Behavioral, and Social Benefits. Journal of Urban Health, 97(4), 529-542. https://doi.org/10.1007/s11524-020-00434-w

Wood, L., Hooper, P., Foster, S., \& Bull, F. (2017). Public green spaces and positive mental health - investigating the relationship between access, quantity and types of parks and mental wellbeing. Health and Place, 48, 63-71. https://doi.org/10.1016/j.healthplace.2017.09.002 


\section{Appendix}

\section{Figure 1}

Study Setting and Park Service Area
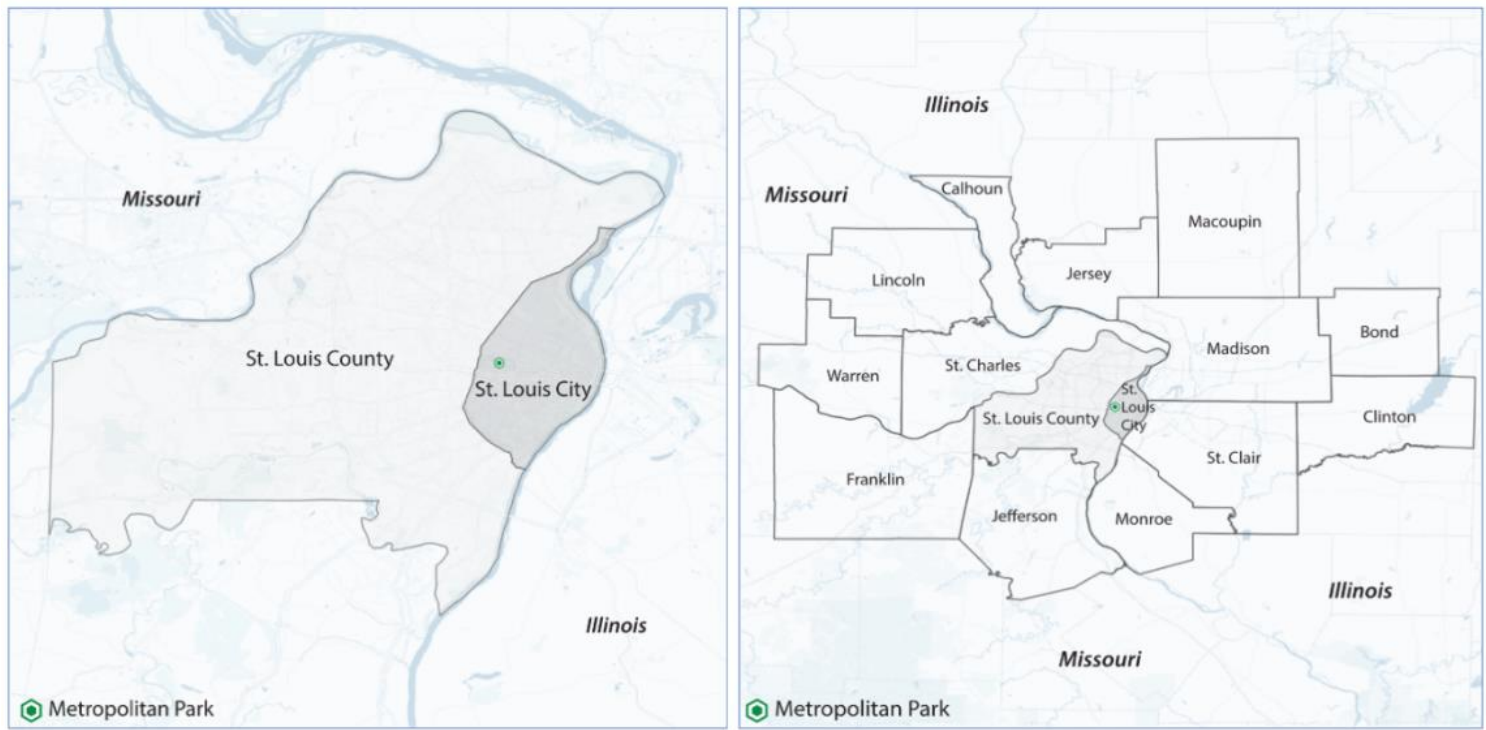

Figure 2

Sample of Voice Data Collected by Citizen Scientists
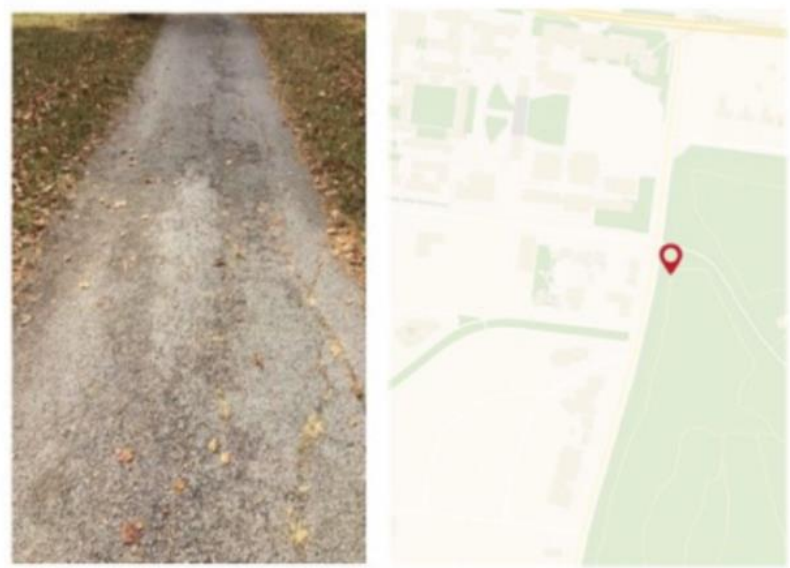

Why did you take this picture?

[ 4 1] : there are a lot of places on the soft path where the finely ground rock has been washed away and never replaced. It's tough for runners especially older ones like me to run on hard surfaces.'

Tags:

Recreation 